\title{
Understanding the Science Behind the Inflammatory Cascade of Dry Eye Disease
}

\author{
Lisa M Nijm ${ }^{12}$ and Grace E Dunbar² \\ 1. Founder and Medical Director, Warrenville EyeCare and LASIK, Cornea, Cataract, and Refractive Surgery, Warrenville, IL, USA; 2. University of Illinois \\ Eye and Ear Infirmary, Department of Ophthalmology and Visual Sciences, Chicago, IL, USA
}

DOI: https://doi.org/10.17925/USOR.2019.12.1.15

$\mathrm{D}$ ry eye disease (DED) affects tens of millions of patients everyday. Bench and clinical research, along with advancements in clinical diagnostic modalities have deepened our appreciation of the heterogeneity of DED and called attention to the inflammatory processes that occur in many patients. This article provides a concise review of the known afferent and efferent pathways of the ocular surface inflammatory response in dry eye disease. Understanding the pathophysiology behind DED will give clinicians a better understanding of this complex disease state and hopefully lead to earlier diagnosis of the condition and improved treatment modalities.

\section{Keywords}

Dry eye disease, ocular surface inflammation, pathophysiology, inflammatory cascade, afferent pathways, efferent pathways in ocular inflammation, hyperosmolar stress, tear film instability, adaptive immune response, conjunctival goblet cell dysfunction, ocular surface homeostasis

Disclosure: Lisa M Nijm and Grace E Dunbar have nothing to disclose in relation to this article.

Review Process: This article is a short opinion piece and has not been submitted to external peer reviewers, but was reviewed by the Editorial Board for accuracy before publication.

Compliance with Ethics: This article is an opinion piece and does not report on new clinical data, or any studies with human or animal subjects performed by the author.

Authorship: All named authors meet the International Committee of Medical Journal Editors (ICMJE) criteria for authorship of this manuscript, take responsibility for the integrity of the work as a whole, and have given final approval to the version to be published.

Received: October 11, 2019

Published Online: April 23, 2019

Citation: US Ophthalmic Review. 2019;12(1):15-6

Corresponding Author: Lisa M Nijm, MD, JD, Warrenville EyeCare \& LASIK, 2S631 State Route 59, Warrenville, IL 60555, USA. E: LMNijm@uic.edu

Support: No funding was received for

the publication of this article.
Dry eye disease (DED) is the one of the leading causes of adult visits to ophthalmologists in the USA, with an estimated prevalence of $5-50 \%$ in adults over the age of 50 years. ' Studies have estimated over 16 million patients have been diagnosed with DED, even in patients as young as 18-34. ${ }^{1}$ As the "Baby Boom" population continues to age, the importance of identifying and diagnosing DED has become paramount. In 2017, the Tear Film \& Ocular Society (TFOS) Dry Eye Workshop (DEWS) Definition and Classification Subcommittee released a revised definition of $\mathrm{DED}_{1}{ }^{2}$ which acknowledges that ocular surface inflammation commonly plays an important role in the pathophysiology of the disease:

"Dry eye is a multifactorial disease of the ocular surface characterized by a loss of homeostasis of the tear film, and accompanied by ocular symptoms, in which tear film instability and hyperosmolarity, ocular surface inflammation and damage, and neurosensory abnormalities play etiological roles."

Bench and clinical research, along with advancements in clinical diagnostic modalities have deepened our appreciation of the heterogeneity of DED and called attention to the inflammatory processes that occur in many patients. ${ }^{2}$ Ancillary testing to aid in the evaluation of inflammation in patients with DED may include conjunctival biopsy, impression cytology, and identification of inflammatory biomarkers such as MMP-9, human leukocyte antigen DR-1 (HLA-DR-1), and intercellular adhesion molecule 1 (ICAM-1) in tear and impression cytology samples. Understanding the normal anatomy of the ocular surface and the effects of the afferent and efferent pathways of DED will give clinicians a better understanding of the current rationale behind diagnostic methods and treatment options for this complex disease state.

\section{Normal anatomy}

The ocular surface is comprised of the cornea, conjunctiva, accessory lacrimal glands, and meibomian glands. The main lacrimal gland, the ocular surface, and their neural interconnections make up the lacrimal functional unit, ${ }^{3}$ and secrete fluids that create a complex tear film to help maintain ocular surface homeostasis. Anti-microbial molecules, immunoglobulins, immunomodulators, and mucins secreted by conjunctival goblet cells are all important tear film constituents which help regulate ocular immune homeostasis. Resident monocytes, macrophages, neutrophils, dendritic cells, Langerhans cells, natural killer cells, regulatory $T$ cells, and effector $T$ cells on the ocular surface also help mediate immune homeostasis. Disruption of any element of the lacrimal functional unit can lead to tear film instability, increased tear film osmolarity, and can trigger an inflammatory response on the ocular surface.

\section{Afferent wing of the ocular surface inflammatory response in dry eye disease}

A variety of extrinsic and intrinsic stressors may disrupt the lacrimal functional unit and lead to tear hyperosmolarity. Potential stressors include low humidity environments, topical medications, mechanical forces, desiccation, infection, aging, and dysfunctional tear secretion. Tear film instability 
and hyperosmolarity can generate an innate immune response, which provides first-line non-specific defense and inflammatory reactions. The initiation of innate immune responses is mediated by pattern-recognition receptors, such as toll-like receptor 4 , which has increased expression in murine models of induced DED. ${ }^{4}$ Receptor recognition triggers activation of inflammasomes, such as NLRP3 and NLRP6, which are activated in human corneal epithelial and murine models of DED, and subsequent secretion of proinflammatory cytokines such as interleukin-1 beta (IL-1ß). ${ }^{5}$

In addition to generating an innate immune response, hyperosmolar stress generates a proinflammatory microenvironment on the ocular surface. In vitro, rat, and murine models have demonstrated that ocular surface hyperosmolar stress activates mitogen activated protein kinases, ${ }^{6,7}$ stimulates the expression of cytokines (IL-6, IL-8, IL-1 $\alpha, \mid \mathrm{L}-1 \beta$, TNF- $\alpha$ ), ${ }^{6-9}$ and increases the production of matrix metalloproteinases (MMP-9, MMP-1, MMP-13, MMP-3).67,10 In vitro studies have also demonstrated that hyperosmolarity can induce apoptosis in cultured corneal epithelial cells, ${ }^{11}$ and experimentally induced DED in murine models has also demonstrated apoptosis of ocular surface tissue cells. ${ }^{12}$

Hyperosmolar stress can trigger an adaptive immune response through overexpression of HLA-DR in the conjunctival epithelium ${ }^{13}$ and increased levels of chemokines such as CCL20, CXCL9, CXCL10, and CXCL11 in the tear film and ocular surface. ${ }^{14,15}$ These chemokines and receptors promote activation of antigen presenting cells, especially corneal dendritic cells, ${ }^{16}$ and recruitment of inflammatory mediators. Increased corneal lymphangiogenesis along with increased honing of antigen presenting cells (APCS) to draining lymph nodes have been observed in murine models of induced DED. ${ }^{17}$ Once they are in draining lymph nodes, APCs then prime naïve T cells, presumably to unknown ocular surface antigens. ${ }^{18}$ Primed effector Th1, Th17, and natural killer (NK) cells travel back to the ocular surface, where they secrete interferon-gamma (IFN $\gamma$ ) and IL-17. ${ }^{19-22}$

\section{Efferent wing of the ocular surface inflammatory response in dry eye disease}

Secretion of IFN $\gamma$ by Th1 and NK cells promotes loss of conjunctival goblet cells, which nurture the tear film through the provision of proteins and mucin. In murine culture models, it has been observed that even low exposure to IFN $\gamma$ results in structural changes in goblet cells and reduced proliferation. ${ }^{23}$ In murine models, exogenous administration of IFN $\gamma$ and induced DED result in decreased goblet cell density. ${ }^{24,25}$ Compared to healthy individuals, subjects with tear dysfunction have been observed to

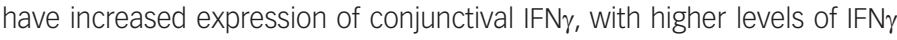
correlating with increased goblet cell loss. ${ }^{26}$ In addition to promoting loss of conjunctival goblet cells, it has been demonstrated in murine models of Sjögren's Syndrome that IFNy also has a role in inducing lacrimal acinar apoptosis through caspase induction. ${ }^{27}$

While IFN $\gamma$ has been implicated in conjunctival goblet cell and lacrimal acinar loss, IL-17 secreted by Th17 cells plays an important role in corneal barrier disruption and induction of matrix metalloproteinase production. ${ }^{21}$ Matrix metalloproteinases are enzymes involved in extracellular matrix degradation and, on the ocular surface epithelium, they promote barrier disruption by destroying tight junctions. Desiccating stress stimulates increased corneal epithelial levels of MMP-1, -3, -9, and -10 in murine models of DED. ${ }^{28}$ MMP-9 has especially been implicated in the pathogenesis of DED as MMP-9 knockout mice are more resistant to corneal epithelial disruption than wildtype controls in models of induced DED. ${ }^{29}$ Investigations using impression cytology have demonstrated that patients with dry eye have significantly higher levels of MMP-9 and IL-17 in the conjunctival epithelium compared to healthy controls. ${ }^{21}$ In murine models, desiccating stress leads to ocular surface infiltration by $T$ cells and increased production of IL-17. Neutralization of IL-17 leads to reduced levels of MMP-9 and MMP-3 in vitro. ${ }^{21}$

\section{Conclusion}

Hyperosmolar stressors and tear film instability trigger the innate immune response and secretion of numerous cytokines to create a vicious cycle that perpetuates the signs and symptoms of DED. Understanding the afferent and efferent pathways of DED will give clinicians a better understanding of this complex disease state and hopefully lead to earlier diagnosis and improved treatment. Application of this greater comprehension of the mechanism of disease to clinical practice will hopefully lead to fewer patients suffering from undiagnosed DED. •
1. Farrand $K F$, Fridman $M$, Stillman IÖ, Schaumberg DA. Prevalence of diagnosed dry eye disease in the United States among adults aged 18 years and older. Am J Ophthalmol. 2017;182:90-8.

2. Craig JP, Nichols KK, AKpek EK, et al. TFOS DEWS II Definition and Classification Report. Ocul Surf. 2017;15:276-83.

3. Stern ME, Gao J, Siemasko KF, et al. The role of the lacrimal functional unit in the pathophysiology of dry eye. Exp Eye Res. 2004:78:409-16.

4. Lee HS, Hattori T, Park EY, et al. Expression of toll-like receptor 4 contributes to corneal inflammation in experimental dry eye disease. Invest Ophthalmol Vis Sci. 2012;53:5632-40.

5. Chi W, Hua X, Chen X, et al. Mitochondrial DNA oxidation induces imbalanced activity of NLRP3/NLRP6 inflammasomes by activation of caspase-8 and BRCC36 in dry eye. J Autoimmun 2017:80:65-76.

6. De Paiva CS, Corrales RM, Villarreal AL, et al. Corticosteroid and doxycycline suppress MMP-9 and inflammatory cytokine expression, MAPK activation in the corneal epithelium in experimental dry eye. Exp Eye Res. 2006;83:526-35.

7. Luo L, Li DQ, Corrales RM, Pflugfelder SC. Hyperosmolar saline is a proinflammatory stress on the mouse ocular surface. Eye Contact Lens. 2005;31:186-93.

8. Higuchi A, Kawakita T, Tsubota K. IL-6 induction in desiccated corneal epithelium in vitro and in vivo. Mol Vis. 2011:17:2400-6.

9. Li DQ, Luo L, Chen Z, et al. JNK and ERK MAP kinases mediate induction of IL-1beta, TNF-alpha and IL-8 following hyperosmolar stress in human limbal epithelial cells. Exp Eye Res. 2006;82:588-96.

10. Li DQ, Chen Z, Song XJ, et al. Stimulation of matrix metalloproteinases by hyperosmolarity via a JNK pathway in human corneal epithelial cells. Invest Ophthalmol Vis SCi. 2004:45:4302-11.
11. Luo L, Li DQ, Pflugfelder SC. Hyperosmolarity-induced apoptosis in human corneal epithelial cells is mediated by cytochrome $\mathrm{c}$ and MAPK pathways. Cornea. 2007;26:452-60.

12. Yeh S, Song XJ, Farley W, et al. Apoptosis of ocular surface cells in experimentally induced dry eye. Invest Ophthalmol Vis SCi. 2003:44:124-9

13. Versura P, Profazio V, Schiavi C, Campos EC. Hyperosmolar stress upregulates HLA-DR expression in human conjunctival epithelium in dry eye patients and in vitro models. Invest Ophthalmol Vis Sci. 2011; 52:5488-96.

14. Yoon KC, Park CS, You IC, et al. Expression of CXCL9, -10, -11, and CXCR3 in the tear film and ocular surface of patients with dry eye syndrome. Invest Ophthalmol Vis Sci. 2010;51:643-50.

15. Choi W, Li Z, Oh HJ, et al. Expression of CCR5 and its ligands CCL3, -4 , and -5 in the tear film and ocular surface of patients with dry eye disease. Curr Eye Res. 2012:37:12-7.

16. Kheirkhah A, Rahimi Darabad R, Cruzat A, et al. Corneal epithelial immune dendritic cell alterations in subtypes of dry eye disease: a pilot in vivo confocal microscopic study. Invest Ophthalmol Vis Sci. 2015;56:7179-85

17. Goyal S, Chauhan SK, El Annan J, et al. Evidence of corneal lymphangiogenesis in dry eye disease: a potential link to adaptive immunity? Arch Ophthalmol. 2010:128:819-24.

18. El Annan J, Chauhan SK, Ecoiffier T, et al. Characterization of effector T cells in dry eye disease. Invest Ophthalmol Vis SCi. 2009;50:3802-7.

19. Niederkorn JY, Stern ME, Pflugfelder SC, et al. Desiccating stress induces T cell-mediated Sjogren's Syndrome-like lacrimal keratoconjunctivitis. J Immunol. 2006;176:3950-7.

20. Stern ME, Gao J, Schwalb TA, et al. Conjunctival T-cell subpopulations in Sjögren's and non-Sjögren's patients with dry eye. Invest Ophthalmol Vis Sci. 2002;43:2609-14.
21. De Paiva CS, Chotikavanich S, Pangelinan SB, et al. IL-17 disrupts corneal barrier following desiccating stress. Mucosal Immunol. 2009;2:243-53.

22. Chen Y, Chauhan SK, Saban DR, et al. Interferon-gamma-secreting NK cells promote induction of dry eye disease. J Leukoc Biol. 2011;89:965-72

23. Coursey TG, Tukler Henriksson J, Barbosa FL, et al. Interferon-gamma-induced unfolded protein response in conjunctival goblet cells as a cause of mucin deficiency in Sjogren syndrome. Am J Pathol. 2016;186:1547-58.

24. De Paiva CS, Villarreal AL, Corrales RM, et al. Dry eye-induced conjunctival epithelial squamous metaplasia is modulated by interferon-gamma. Invest Ophthalmol Vis Sci. 2007;48:2553-60.

25. Zhang X, Chen W, De Paiva CS, et al. Interferon-gamma exacerbates dry eye-induced apoptosis in conjunctiva through dual apoptotic pathways. Invest Ophthalmol Vis Sci. 2011:52:6279-85.

26. Pflugfelder SC, De Paiva CS, Moore QL, et al. Aqueous tear deficiency increases conjunctival interferon-gamma (IFN-gamma) expression and goblet cell loss. Invest Ophthalmol Vis SCi. 2015;56:7545-50.

27. Bian F, Barbosa FL, Corrales RM, et al. Altered balance of interleukin-13/interferon-gamma contributes to lacrimal gland destruction and secretory dysfunction in CD25 knockout mode of Sjogren's syndrome. Arthritis Res Ther. 2015;17:53.

28. Corrales RM, Stern ME, De Paiva CS, et al. Desiccating stress stimulates expression of matrix metalloproteinases by the corneal epithelium. Invest Ophthalmol Vis Sci. 2006:47:3293-302

29. Pflugfelder SC, Farley W, Luo L, et al. Matrix metalloproteinase-9 knockout confers resistance to corneal epithelial barrier disruption in experimental dry eye. Am J Pathol. 2005;166:61-71. 